\title{
Experience of intermittent epidural analgesia for open liver resection
}

\author{
Takashi Matsusaki*, Ryuji Kaku, Daisuke Ono, Arata Taniguchi and Hiroshi Morimatsu \\ Department of Anesthesiology and Resuscitology, Okayama University Hospital, Okayama, Japan
}

\begin{abstract}
There were some reports regarding intermittent method of epidural analgesia would be better in terms of postoperative analgesia with the obstetric analgesia for labors.

In this study, the main outcome was to investigate the appropriate dosage of intermittent bolus injection from epidural block $(0.2 \%$ ropivacaine $200 \mathrm{~mL}+\mathrm{fentanyl}$ $200-400 \mu \mathrm{g} / \mathrm{kg}$ ). The definition of "Appropriate" was we had no side effects such as neurologic complication such as numbing and hypotension, and to keep the patient's good pain control controlled below resting Numerous Rating Scale (NRS) three until 24 hours after surgery (ICU periods). This study started from 2 ml$6 \mathrm{ml} / 60$ minutes (minimum dose) according to our protocol. In $2 \mathrm{ml}$ protocol, one patient cleared our protocol, however two patients couldn't clear in terms of NRS. In $3 \mathrm{ml}$ protocol, we could keep the good pain control based on the definition of "effectiveness" (NRS, bolus request, the necessity of increased pain analgegics and no deleterious events).

$3 \mathrm{~mL}$ of intermittent epidural contrast for the open hepatectomy was identified by our clinical study in order to control the postoperative pain. The results of our study might contribute to future research regarding intermittent epidural administration, as well as provide patients with more excellent analgesia.
\end{abstract}

Abbreviations: PIB: programmed intermittent bolus; NRS: numerous rating scale; BMI: body mass index

\section{Introduction}

Epidural analgesia has been a milestone of postoperative pain management for the patients with open liver resection compared to other methods such as intravenous opioid [1,2]. We have two methods of intermittent bolus or continuous one regarding epidural analgesia for the patients with open liver resection surgery. The problem of continuous method might tend to be less effective with longer usage [3]. There were some reports regarding intermittent method of epidural analgesia would be better in terms of postoperative analgesia with the obstetric analgesia for labor [4]. There were no reports regarding the intermittent epidural analgesia for the patients with open liver resection especially in during the postoperative period.

The objectives of this research was to clarify the appropriate bolus dosage of intermittent epidural analgesia for open liver resection.

\section{Materials and methods}

This prospective invasive clinical research was conducted at our tertiary teaching hospital in Japan with institutional review board approval of Okayama University Graduate School of Medicine, Dentistry and Pharmaceutical Sciences, Ethics Committee (Number: 05002) and after obtaining the patients' written informed consent for study participation. This study complied with the principles of the Declaration of Helsinki.

Patients who had received liver resection according to HCC and metastatic tumor performed under epidural analgesia, were included. Only conscious patients with whom we would be able to converse were included. The age of patients was over 20 until 80 years-old.
The patients with difficult epidural insertion, allergies to regional anesthetics, renal dysfunction (Serum creatinine $2.0 \mathrm{mg} \mathrm{dL}^{-1}$ ), liver dysfunction (Serum AST/ALT $>100 \mathrm{mg} \mathrm{dL}^{-1}$ ), severe cardiac dysfunction (ejection fraction $<40 \%$ ), convulsions, severe asthma, and the unpredicted altered consciousness such as delirium whom we couldn't assess NRS were excluded from the study.

The main outcome was to investigate the appropriate dosage of intermittent bolus injection from epidural block. The definition of "Appropriate" was we had no side effects such as neurologic complication such as numbing and hypotension, and to keep the patient's good pain control controlled below resting Numerous Rating Scale (NRS) three. The definition of "effectiveness" was to keep the good control of NRS at rest and the definition of "ineffectiveness" was 1 . NRS $\geqq 4$ even by using other analgesics such as acetaminophen, flurbiprofen axetil and dexmedetomidine). 2. The frequency of bolus per hour was over 20. 3. The timing of when the other intensivists except for the researchers thought the necessary bolus to improve the analgesic effects by the poor spread of the epidural analgesics. 4 . The occurrence of the deleterious effects by PIB: Hypotension <systolic arterial pressure $60 \mathrm{mmHg}$, bradycardia: below 40 heart rate, Numbness and paralysis of lower legs.

The second outcome was to collect the information regarding programmed intermittent bolus (PIB) pump of $\mathrm{CADD}^{\circ}$-Solis PIB

*Correspondence to: Takashi Matsusaki, Department of Anesthesiology and Resuscitology Okayama University Graduate School of Medicine, Dentistry, and Pharmaceutical Sciences, 2-5-1 Shikata-cho, Kita-ku, Okayama 700-8558, Japan, E-mail: matusakik@ybb.ne.jp

Key words: numerous rating scale, intermittent epidural block, open hepatectomy

Received: September 30, 2019; Accepted: October 10, 2019; Published: October 14,2019 
pimp (Patient Controlled Epidural Analgesia), usage of analgesia and frequency of bolus and request (intermittent: $2-6 \mathrm{ml} / \mathrm{hr}$, bolus $2 \mathrm{ml}$, lockout 15 minutes/3 times/hr). The information of other modality acetaminophen, flurbiprofen axetil and dexmedetomidine were also collected. An individual nurse will try to collect the patient's data every two hours except for at the timing of sleeping. The study period was from the admission of ICU until the discharge of the ICU (Postoperative day 1)

According to the anti-cancer therapy protocol, the required sample size in this study was 3-30 patients [5]. This showed that if first dose would be successful, this study was done. Unless only one patient would be successful, another case will continue using same dose. If three cases would be successful, this dose was "appropriate". Unless even one of this dose would be successful, we will go to next step. Since we did not know what dose of intermittent epidural injection would be appropriate for continuous administration, we created a new protocol for this research, keeping the concentration of regional analgegics at the lowest dosage possible for patient safety. We started from $2 \mathrm{~mL}$ until $6 \mathrm{~mL}$. If even $6 \mathrm{~mL}$ would be unsuccessful, we can't find any appropriate dose as conclusion.

We used the pump of CADD'-Solis PIB as Patient controlled epidural analgesia since the end of surgery. The medicine was $0.2 \%$

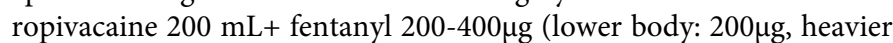
body: $400 \mu \mathrm{g}$ ). This study started from $2 \mathrm{ml} / 60$ minutes (minimum dose) according to our protocol.

\section{Anaesthesia procedure}

The epidural block's indication was the patients without any thrombocytopenia (Platelet $>100000 / \mu \mathrm{L}$ ) and PT-INR $<1.5$ based on the ASRA's guideline [6].

An epidural catheter was inserted at Th7/8 or $8 / 9$ preoperatively in the Operating room with the patient in the lateral position. The appropriate insertion site was decided by the attending anaesthesiologist. Accurate placement of the needle (TUOHY EPIDURAL NEEDLE BY BRAUN (BMG332186) NEEDLE, 18G. X 4.75 TUOHY.) into the epidural space was confirmed by the traditional method of "loss of resistance" to the injection of saline. After the epidural catheter was inserted via the needle in a cephalad direction, $1 \%$ lidocaine with epinephrine (1:50000) (4ml) was injected epidurally in order to exclude inadvertent intravenous insertion Thereafter, the catheter was fixed at a depth of $3-5 \mathrm{~cm}$ from the insertion site using sterile tape. Intraoperatively we used epidural block continuously or intermittently according to the individual anesthesiologists without limitation. Intraoperative final amount of remifentanil, fentanyl and $0.2 \%$ ropivacain's total dose were recorded. The induction and maintenance of anesthesia was Total Intravenous Anesthesia (propofol) or inhalation (Desflurane or Sevoflurane). We extubated at the operating room and started PCEA.

After the admission of ICU we started our clinical study. We have collected NRS at rest every two hours by nurse unrelated to study or physician related to study. Our goal was to control the pain of below $4 / 10$. If the patient felt pain and required further analgesia, analgesics were given according to our protocol for analgesia management postoperatively. 1. The usage of PCEA bolus. 2. The usage of IVAcetoaminophen $(15 \mathrm{mg} / \mathrm{kg})$ at least every six hours. 3. The usage of IV-Non-Steroidal Anti-Inflammatory Drugs (flurbiprofen axetil) at least every 12 hours. 4 . The usage of IV-continuous dexmedetomidin $(0.2-1 \mathrm{ng} / \mathrm{kg} / \mathrm{hr}$ ). We have also collected side effect (nausea/vomiting, itching) related to PCEA and deleterious effect (hypotension, bradycardia and numbness of leg) by nurse unrelated to study or physician related to study.

\section{Surgical procedure}

The type of laparotomy was decided on according to the type of partial hepatectomy and the patient's constitution. Preoperative cholangiography was not usually performed. All liver resection was performed using Pringle technique and the shape of the wound was reverese-Mercedes Bentz (bilateral subcostal incision). One or two drainage tubes were inserted into the abdominal space. The surgical time, estimated blood loss, transfusion amounts and fluids were also collected. The bilateral subcostal approach, extended to the right as far as the midaxillary line, to the left as far as the lateral border of the rectus muscle and in the midline superiorly to the xiphoid process, is the classical approach for major hepatectomies and for liver transplants [7].

\section{Results}

Case 1: A 55-year-old man (height $172 \mathrm{~cm}$, body weight $78 \mathrm{~kg}$, BMI 27) had a history of metastatic liver tumor with hypertension. The posterior resection of liver was scheduled under epidural anaesthesia, which was administered at the Th8/9 level. Intraoperatively his epidural $0.2 \%$ ropivacaine $33 \mathrm{~mL}$ was used with IV-fentanyl $100 \mu \mathrm{g}$ and anesthesia maintenance was conducted by AOS-Remifentanil. He was extubated in OR with shivering, however his pain was controlled successfully $<\mathrm{NRS} 4 / 10$, although he used PCEA bolus and IV-acetoamiphen once during ICU stay.

Case 2: A 59-year-old man (height $166 \mathrm{~cm}$, body weight $64 \mathrm{~kg}$, BMI 23) had a history of metastatic liver tumor with hypertension. The internal resection of liver was scheduled under epidural anaesthesia, which was administered at the Th8/9 level and anesthesia maintenance was conducted by TIVA. His epidural $0.2 \%$ ropivacaine $28 \mathrm{~mL}$ was used

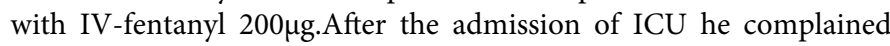
the abdominal pain, so the rescue-bolus of epidural (PCEA) and IVacetoaminophen were given to him. Unfortunately we couldn't control his pain NRS $<4 / 10$ during ICU stay. He also complained back pain and right shoulder pain, however he didn't want to have other analgesics regardless of his higher pain score.

Case 3: A 80-year-old man (height $167 \mathrm{~cm}$, body weight $62 \mathrm{~kg}$, BMI 22) had a history of HCC with hypertension and COPD. The posterior resection of liver was scheduled under epidural anaesthesia, which was administered at the $\mathrm{Th} 7 / 8$ level. His epidural $0.2 \%$ ropivacaine $37 \mathrm{~mL}$ was used with IV-fentanyl $500 \mu \mathrm{g}$. His anesthesia maintenance was conducted by AOD-Remifentanil. At the immediate timing and 2 hours after the admission of ICU he complained abdominal using IV-acetoaminophen and bolus of PCEA, however we couldn't control his pain. So According to our protocol, the intermittent dose $(2 \mathrm{~mL})$ couldn't satisfy our criteria (NRS $<4 / 10)$. Only case 1 was satisfied with our criteria. Next study, we increased the intermittent dose $3 \mathrm{~mL}$ every one hour.

Case 4: A 73-year-old female (height $135 \mathrm{~cm}$, body weight $44 \mathrm{~kg}$, BMI 25) had a history of HCC with diabetes mellidus. The subsequent resection of liver was scheduled under epidural anaesthesia, which was administered at the Th8/9 level. Intraoperatively his epidural $0.2 \%$ ropivacaine $18 \mathrm{~mL}$ was used with IV-fentanyl $300 \mu \mathrm{g}$ and anesthesia maintenance was conducted by AOS-Remifentanil. She needed IVacetoaminophen after the admission of ICU, however her pain control was controlled successfully (NRS $<4 / 10)$. 
Case 5: A 70-year-old man (height $174 \mathrm{~cm}$, body weight $76 \mathrm{~kg}$, BMI 23) had a history of HCC with hypertension. The posterior resection of liver was scheduled under epidural anaesthesia, which was administered at the Th7/8 level. Intraoperatively his epidural $0.2 \%$ ropivacaine $38 \mathrm{~mL}$ was used with IV-fentanyl $400 \mu \mathrm{g}$ and anesthesia maintenance was conducted by TIVA. He needed IV-acetoaminophen at 8 hours after the admission of ICU and often used bolus of PCEA, however his pain control was controlled successfully (NRS $<4 / 10$ ).

Case 6: A 70-year-old man (height $164 \mathrm{~cm}$, body weight $53 \mathrm{~kg}$, BMI 20) had a history of HCC with hypertension and diabetus mellidus. The internal resection of liver was scheduled under epidural anaesthesia, which was administered at the Th8/9 level. Intraoperatively his epidural $0.2 \%$ ropivacaine $21 \mathrm{~mL}$ was used with IV-fentanyl $250 \mu \mathrm{g}$ and anesthesia maintenance was conducted by AOD-Remifentanil. He needed IV-acetoaminophen after the admission of ICU, however his pain control was good controlled successfully $(\mathrm{NRS}<4 / 10)$.

All patients didn't have over-requested frequency for PCEA, any severe hypotension and neurologic problems related to epidural block. Finally we could keep the good pain control based on the definition of "effectiveness" (NRS, bolus request, the necessity of increased pain analgegics and no deleterious events) (Table 1).

So $3 \mathrm{~mL}$ dose/hour of intermittent epidural injection would be appropriate without any problems.

\section{Discussion}

We found that $3 \mathrm{~mL}$ of intermittent epidural block ( $0.2 \%$ ropivacain) would be feasible in terms of pain control and deleterious effects for the open hepatectomy. There were no reports regarding the intermittent injection for epidural block postoperatively. The background of "appropriate dose" was well controlled analgesia (NRS: within 3/10) and safety's issue (no severe systolic hypotension $<60 \mathrm{mmHg}$ related to PIB infusion and neurological complication). We cited the anti-cancer drug's Phase 1 protocol and tried to identify the appropriate dose [5]. The safety's information regarding PIB pump was given to us, however in our hospital this PIB pump was used for the first time.

There were some reports regarding epidural intermittent injection compared to continuous one. Some reports showed that epidural intermittent injection had been more excellent in terms of the pain intensity, satisfaction and less side effect (numbness of lower legs) in the delivery $[4,8,9]$. In the knee surgery, epidural intermittent injection would be feasible and safe in addition to the analgesic effect. The side effect related to epidural block was the minimal compared to continuous block [10]. The postoperative analgesia regarding open liver resection was conducted with regional analgesia (including epidural analgesia, intrathecal analgesia, paravertebral block and transversus abdominis plane), local-inlation near the surgical site and IV-opioid [11-13]. Hughe $M$, et al. showed that epidurals analgesia provided superior pain relief to alternatives (IV-opioid, paracetamol, non-steroid inflammatory drug and local filtration) but this does not translate into reduced length of stay or complication rate following liver surgery [2]. Ganapathi S, et al. also showed that continuous epidural Bupivacaine $(0.1 \%)$ with fentanyl $(2 \mu \mathrm{g} / \mathrm{mL})$ infusions enabled 0ne-third $(22 / 66)$ of patients 0-pain [14]. In our institution, epidural analgesia was the standard unless contraindication, however we didn't have any definite guideline for the pain management regarding open hepatectomy. The merit for the intermittent injection compared to continuous one would be reported as that low dose's regional anlgesics secured same analgesic

Table 1. Summary of patient characteristics

\begin{tabular}{|c|c|c|c|c|c|c|}
\hline & Case1 & Case2 & Case3 & Case4 & Case5 & Case6 \\
\hline Intermittent Dose/hr (mL) & 2 & 2 & 2 & 3 & 3 & 3 \\
\hline Age/Gender & $55 / \mathrm{M}$ & $59 / \mathrm{M}$ & $80 / \mathrm{M}$ & $73 / \mathrm{F}$ & $70 / \mathrm{M}$ & $70 / \mathrm{M}$ \\
\hline BMI & 27 & 23 & 22 & 25 & 23 & 20 \\
\hline Disease & Metastatic Tumor & Metastatic Tumor & $\mathrm{HCC}$ & $\mathrm{HCC}$ & $\mathrm{HCC}$ & $\mathrm{HCC}$ \\
\hline Liver resected Area & Posterior & Internal & Posterior & subsequent & Posterior & Internal \\
\hline ASA & 2 & 2 & 3 & 2 & 2 & 3 \\
\hline Anesthesia (TIVA or VIMA) & VIMA & TIVA & VIMA & VIMA & TIVA & VIMA \\
\hline Epidural insertion & Th8/9 & Th8/9 & Th7/8 & Th8/9 & $\mathrm{Th} 7 / 8$ & $\mathrm{Th} 8 / 9$ \\
\hline Surgical Time & $7: 15$ & $5: 35$ & $5: 30$ & 4:06 & $3: 50$ & $3: 43$ \\
\hline Estimated Blood loss (mL) & 2535 & 1700 & 555 & 190 & 50 & 100 \\
\hline Transfusion & Yes & Yes & Yes & Yes & No & No \\
\hline Fentanyl $(\mu \mathrm{g})$ & 100 & 200 & 500 & 300 & 400 & 250 \\
\hline Remifentanil $(\mu \mathrm{g})$ & 2000 & 2621 & 2464 & 1618 & 2877 & 825 \\
\hline Intraoperative total of $0.2 \%$ Ropivacaine $(\mathrm{mL})$ & 33 & 28 & 37 & 18 & 38 & 21 \\
\hline Intermittent Dose/hr (mL) & 2 & 2 & 2 & 3 & 3 & 3 \\
\hline Shivering & Yes & No & Yes & Yes & No & Yes \\
\hline Nausea/Vomitting & No & No & Yes & No & No & No \\
\hline Itching & No & No & No & No & No & No \\
\hline Rescue-Acetoaminophen & Yes & Yes & Yes & Yes & Yes & No \\
\hline Rescue-NSAID & No & No & No & No & No & No \\
\hline Rescue-Dexmedetomidin & No & No & No & No & No & No \\
\hline Deleterious effect & No & No & No & No & No & No \\
\hline PIB usage (ml) & 44 & 36 & 48 & 48 & 58 & 52 \\
\hline Bolus frequency & 10 & 6 & 12 & 5 & 13 & 2 \\
\hline Bolus Request & 14 & 6 & 12 & 6 & 15 & 4 \\
\hline NRS criteria & $\circ$ & $x$ & $x$ & $\circ$ & $\circ$ & $\circ$ \\
\hline Final Decision & $\circ$ & $x$ & $x$ & o & $\circ$ & $\circ$ \\
\hline
\end{tabular}

Abbreviation: Gender; M:Male, F: Female, BMI: Body Mass Index, HCC: Hepatic Cell Carcinoma, ASA: American Society of Anesthesia, TIVA: Total intravenous anesthesia, VIMA: Volatile Induction and Maintenance Anesthesia 
effect through intermittent injection in delivery's pain management [4]. That's why we thought lower regional analgesics would contribute to less neurological complication.

In $2 \mathrm{ml}$ protocol one patient could clear our criteria, however two patients couldn't pass NRS criteria. To keep good NRS was difficult by even using bolus injection using patient controlled analgesia's system and other analgesics (acetoaminophen). We assessed $2 \mathrm{ml}$ didn't spread enough to keep good analgesia. We also conducted cold test and this result was consistent with controlled pain area. In $3 \mathrm{ml}$ protocol, we could successfully satisfied with our protocol using PCEA bolus and acetoaminophen. We also confirmed cold test, which was consistent with controlled pain area.

\section{Limitation}

There were some limitation regarding our research. First, the confirmation of epidural space was not conducted by using contrast, but by traditional method (no resistance for pushing saline). Second, the problems regarding postoperative assessment for NRS was considered because some patients had postoperative delirium after liver lisection, especially in older patients [15]. Our patients would have no delirium during these periods fortunately. The third, we examined these assessment during only ICU periods (until 12 hours after surgery). We should have PIB method during at least 48 hours because epidural catheter was removed by 2-4 days after surgery in most of other hospital [16]. The forth, the protocol's validity was discussed. We didn't have any experience using PIB after open-abdominal surgery. That's why we took advantage this Phase 1 study for anti-cancer drugs.

\section{Conclusion}

$3 \mathrm{~mL}$ of intermittent epidural injection for the open hepatectomy was identified by our clinical study in order to control the postoperative pain. The results of our study might contribute to future research regarding intermittent epidural administration, as well as provide patients with more excellent analgesia.

\section{Conflict of interest}

The authors declare no conflict of interest related to this work.

\section{Funding}

Financial support for this work was provided solely by the institutions and departments named in the author affiliations.

\section{Acknowledgement}

The authors thank Aya Ono MD, PhD and Shiro Hinotsu MD, PhD (New Clinical Development of Okayama University Hospital) for their assistance in the design of our research. We would not have been able to complete this research without support of Yuko Mihara (Research Nurse)

\section{Disclosure}

The abstract of this paper was presented at the $112^{\text {th }}$ Annual Meeting of the American Sociological Association as a poster presentation.

\section{References}

1. Hughes MJ, Ventham NT, McNally S, Harrison E, Wigmore S (2014) Analgesia after open abdominal surgery in the setting of enhanced recovery surgery: a systematic review and meta-analysis. JAMA Surg 149: 1224-1230. [Crossref]

2. Hughes M, McNally S, McKeown DW, Wigmore S (2015) Effect of analgesic modality on outcome following open liver surgery: a systematic review of postoperative analgesia. Minerva Anestesiol 81: 541-556. [Crossref]

3. Kaynar AM, Shankar KB (1999) Epidural infusion: continuous or bolus? Anesth Analg 89: 534.

4. George RB, Allen TK, Habib AS (2013) Intermittent epidural bolus compared with continuous epidural infusions for labor analgesia: a systematic review and metaanalysis. Anesth Analg 116: 133-144.

5. Newell DR, Silvester J, McDowell C, Burtles SS (2004) Cancer Research UK. The Cancer Research UK experience of pre-clinical toxicology studies to support early clinical trials with novel cancer therapies. Eur J Cancer 40: 899-906.

6. Horlocker TT, Wedel DJ, Rowlingson JC, Enneking FK, Kopp SL, et al. (2010) Regional anesthesia in the patient receiving antithrombotic or thrombolytic therapy: American Society of Regional Anesthesia and Pain Medicine Evidence-Based Guidelines (Third Edition). Reg Anesth Pain Med 35: 64-101. [Crossref]

7. Sadamori H, Yagi T, Shinoura S, Umeda Y, Yoshida R, et al. (2013) Risk factors for major morbidity after liver resection for hepatocellular carcinoma. Br J Surg 100: $122-129$

8. Tien M, Allen TK, Mauritz A, Habib AS (2016) A retrospective comparison of programmed intermittent epidural bolus with continuous epidural infusion for maintenance of labor analgesia. Curr Med Res Opin 32: 1435-1440. [Crossref]

9. Carvalho B, George RB, Cobb B, McKenzie C, Riley ET (2016) Implementation of Programmed Intermittent Epidural Bolus for the Maintenance of Labor Analgesia. Anesth Analg p. 123.

10. Kang S, Jeon S, Choe JH, Bang SR, Lee KH (2013) Comparison of analgesic effects of programmed intermittent epidural bolus and continuous epidural infusion after total knee arthroplasty. Korean J Anesthesiol 65: S130-S131. [Crossref]

11. Kasivisvanathan R, Abbassi-Ghadi N, Prout J, Clevenger B, Fusai GK, et al. (201) A prospective cohort study of intrathecal versus epidural analgesia for patients undergoing hepatic resection. HPB (Oxford) 16: 768-775. [Crossref]

12. Schreiber KL, Chelly JE, Lang RS, Abuelkasem E, Geller DA, et al. (2016) Epidural Versus Paravertebral Nerve Block for Postoperative Analgesia in Patients Undergoing Open Liver Resection: A Randomized Clinical Trial. Reg Anesth Pain Med 41: 460-468.

13. Bell R, Pandanaboyana S, Prasad KR (2015) Epidural versus local anaesthetic infiltration via wound catheters in open liver resection: a meta-analysis. ANZ J Surg 85: 16-21. [Crossref]

14. Ganapathi S, Roberts G, Mogford S, Bahlmann B, Ateleanu B, et al. (2015) Epidural analgesia provides effective pain relief in patients undergoing open liver surgery. $\mathrm{Br} J$ Pain 9: 78-85. [Crossref]

15. Yoshimura Y, Kubo S, Shirata K, Hirohashi K, Tanaka H, et al. (2004) Risk factors for postoperative delirium after liver resection for hepatocellular carcinoma. World $J$ Surg 28: 982-986.

16. Stamenkovic DM, Jankovic ZB, Toogood GJ, Lodge JP, Bellamy MC (2011) Epidural analgesia and liver resection: postoperative coagulation disorders and epiduralcatheter removal. Minerva Anestesiol 77: 671-679.

Copyright: (2019 Matsusaki T. This is an open-access article distributed under the terms of the Creative Commons Attribution License, which permits unrestricted use, distribution, and reproduction in any medium, provided the original author and source are credited. 